\title{
99. UNA NUEVA SUBESPECIE DE MORICANDIA MORICANDIOIDES (BOISS.) HEYWOOD (BRASSICACEAE).
}

\author{
Pedro SÁNCHEZ GÓMEZ, Miguel Ángel CARRIÓN VILCHES, \\ Antonio HERNÁNDEZ GONZÁLEZ \& Juan GUERRA
}

A new subspecies of Moricandia moricandioides (Boiss.) Heywood (Brassicaceae).

Palabras clave: Moricandia, Brassicaceae, Murcia, España.

Key words: Moricandia, Brassicaceae, Murcia, Spain.

Con motivo de la realización de un proyecto sobre la flora protegida de la Región de Murcia, se revisó el material referente a Moricandia foetida Bourg. ex Coss. incluído en el herbario MUB, encontrándose que el único pliego recolectado (Puerto del Garruchal, Murcia) presentaba el hábito de $M$. foetida, pero caracteres diagnósticos de Moricandia moricandioides (Boiss.) Heywood; sin embargo, no correspondía a ninguna de las subespecies descritas hasta el momento para esta especie (cf. Sobrino, 1993).

Este material parece concordante con lo expuesto por Heywood (1964), que indicó como ciertas poblaciones murcianas presentan caracteres intermedios entre $M$. foetida y $M$. moricandioides, lo cual nos indujo a suponer la existencia de poblaciones producto de la hibridación de estos dos taxones. Sobrino (1997) a partir de estudios de hibridación en el género Moricandia DC. concluye, sin embargo, que es poco probable la existencia de híbridos entre $M$. foetida y $M$. moricandioides.

Teniendo en cuenta los datos anteriores, y tras la recolección durante los años 2000 y 2001 de material con dificultades de adscripción, procedente de la localidad mencionada, se procedió al estudio morfométrico y cualitativo de los caracteres más significativos (Tabla 1). Los resultados obtenidos nos inducen a describir un nuevo taxon como subespecie de M. moricandioides.

Moricandia moricandioides subsp. pseudofoetida Sánchez Gómez, Carrión Vilches, Hernández \& J. Guerra subsp. nov.

A Moricandia moricandioides differt petalis albidis et minoribus, inflorescentia frecuenter flexuosa.

Typus: España, Murcia, Puerto del Garruchal, 30SXH70, 120 m, taludes margosos, 20-III-2001, Sánchez Gómez, Hernández \& Jiménez. MGC 49454. Isotypus: MGC 49453, Syntypus: MGC 49455.

\section{Ic.: Figura 1.}

Planta anual o bisanual, glabra. Tallos de hasta $44 \mathrm{~cm}$, erectos. Hojas basales no arrosetadas, de obovadas a espatuladas, obtusas; margen crenado a lobulado; base atenuada. Hojas caulinares amplexicaules,

Trabajo subvencionado con cargo al proyecto 1FD97-0563 de la DGES y Convenio "Conservación y manejo de los hábitats naturales en relación con el mantenimiento de la biodiversidad en la región de Murcia", con la Consejería de Agricultura, Agua y Medio Ambiente de la Región de Murcia. 


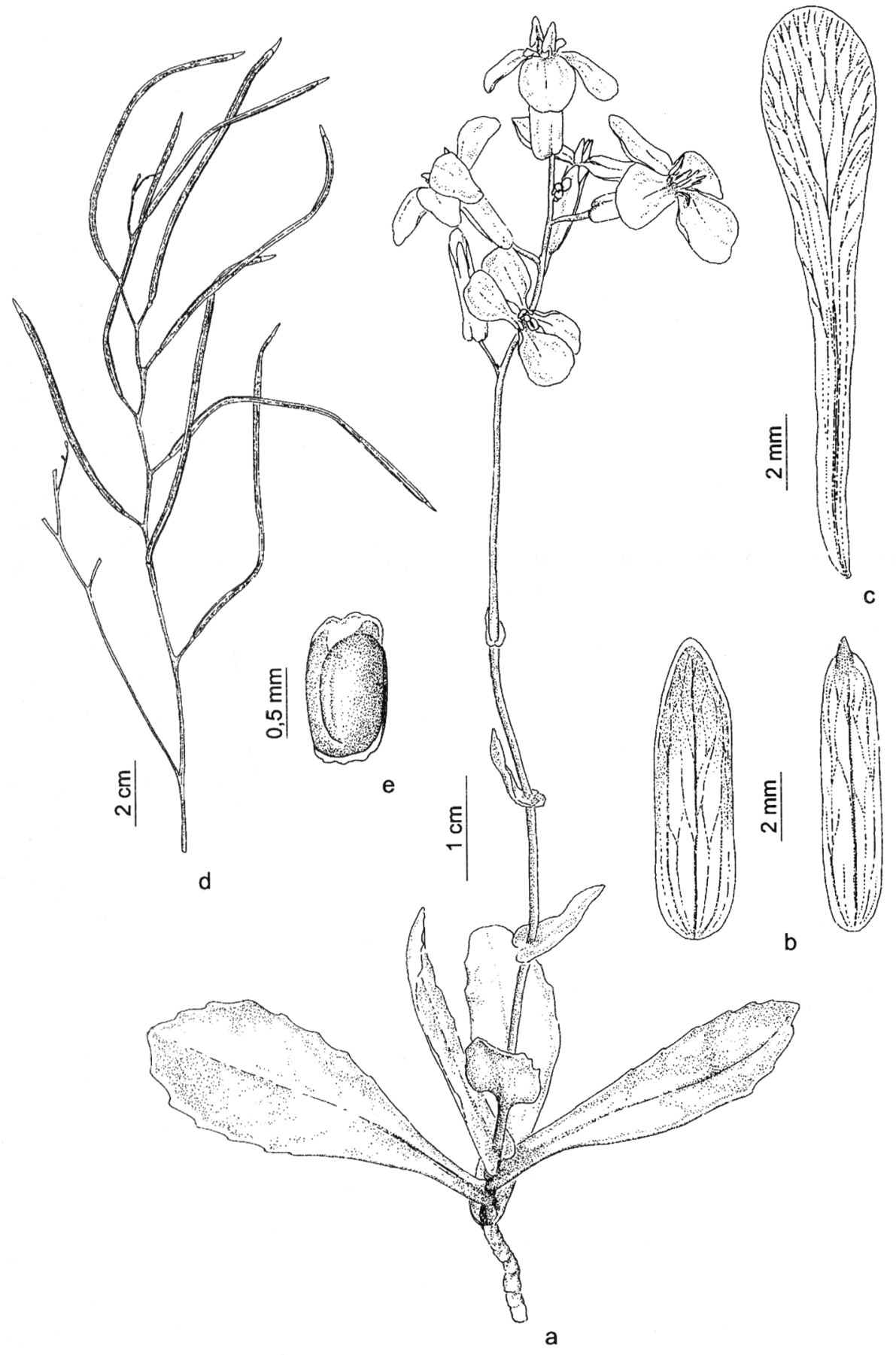

Figura 1. Moricandia moricandioides subsp. pseudo-foetida (MGC 49455). a: hábito; b: sépalos; c: pétalo; (MGC 49456) d: silicuas en racimo flexuoso; e: semilla. 


\begin{tabular}{|c|c|c|c|c|}
\hline \multicolumn{3}{|c|}{$\begin{array}{l}\text { Moricandia moricandioides } \\
\text { subsp. moricandioides }\end{array}$} & \multirow{2}{*}{$\begin{array}{c}\text { Moricandia moricandioides } \\
\text { subsp. pseudo-foetida }\end{array}$} & \multirow{2}{*}{$\begin{array}{l}\text { Moricandia foetida } \\
\text { (20)21-28(31) }\end{array}$} \\
\hline Altura planta $(\mathrm{cm})$ & & $(28) 33-58(80)$ & & \\
\hline Hojas caulinares su & eriores & Agudas a obtusas & Agudas & Agudas \\
\hline Inflorescencia & $\begin{array}{l}\text { Eje } \\
\mathrm{N}^{\circ} \text { de flores }\end{array}$ & $\begin{array}{l}\text { No flexuoso } \\
\text { (10)22-30(56) }\end{array}$ & $\begin{array}{l}\text { Frecuentemente flexuoso } \\
\text { (4)9-11(21) }\end{array}$ & $\begin{array}{l}\text { Frecuentemente flexuoso } \\
\text { (6)11-12(19) }\end{array}$ \\
\hline Pétalos & $\begin{array}{l}\text { Longitud (mm) } \\
\text { Coloración }\end{array}$ & $\begin{array}{c}(17,5) 18,6-20,1(22,5) \\
\text { Purpúreo-violáceos }\end{array}$ & $\begin{array}{l}(15,6) 17,4-18,9(21,5) \\
\text { Blanco-violáceos }\end{array}$ & $\begin{array}{l}(10,8) 13,1-14,7(17,3) \\
\text { Blanco-violáceos }\end{array}$ \\
\hline Sépalos Medianos & & Corniculados & Corniculados & Obtusos \\
\hline Fruto & $\begin{array}{l}\text { Longitud (mm) } \\
\text { Anchura }(\mathrm{mm})\end{array}$ & $\begin{array}{c}70-110(130) \\
1,3-1,5\end{array}$ & $\begin{array}{c}(43,2) 66,7-86,8(105) \\
(1,4) 1,9-2,2(2,5)\end{array}$ & $\begin{array}{c}(47,7) 52,6-60,6(65,5) \\
(1,1) 1,6-2,1(2,4)\end{array}$ \\
\hline Pedicelo & Longitud (mm) & $7,1-7,8$ & $(7) 7,8-9,1(10,9)$ & $(8,2) 9,8-12,3(14,4)$ \\
\hline
\end{tabular}

Tabla 1. Caracteres diferenciales entre Moricandia moricandioides subsp. moricandioides, $M$. moricandioides subsp. pseudo-foetida y $M$. foetida.

lanceoladas, agudas. Inflorescencia racemosa, con el eje frecuentemente flexuoso, (4)9-11(21) flores. Sépalos laterales $10 \times 4 \mathrm{~mm}$, gibosos. Sépalos medianos $10,5 \times 3,5 \mathrm{~mm}$, corniculados. Pétalos (15)17-19(22) mm, estrechados en una uña, blanco-violáceos. Fruto en silícua, (43)67$87(105) \times(1.5) 2(2.5) \mathrm{mm}$, erecto-patentes. Pedicelos (7)8-9(11) x 0,5-1,5 mm. Semillas uniseriadas, 1,6-2,0 $\mathrm{mm}$ de longitud, con ala marginal estrecha.

La nueva subespecie se diferencia, de las restantes conocidas, por presentar menor número de flores por inflorescencia, silicuas más cortas y anchas, pedicelos más largos y pétalos más cortos, así como por la tonalidad más pálida de los pétalos. Se diferencia de $M$. foetida por presentar los sépalos medianos corniculados, lo que confiere un aspecto característico a los botones florales de $M$. moricandioides (Bolós, 1948).

Se encuentra en taludes margosos junto a Moricandia arvensis (L.) DC., Guiraoa arvensis Coss., Reseda lanceolata Lag., etc., entre 100 y $200 \mathrm{~m}$ de altitud, dentro del piso termomediterráneo y ombrotipo semiárido (Sánchez Gómez et. al., 1998).

Hasta el momento tan solo se conoce una población diseminada en $2 \mathrm{~km}^{2}$, por lo que se ha incluido en la categoría de Vulnerable, dentro del Catálogo Regional de Flora Protegida, de inminente aprobación.

\section{MATERIAL ESTUDIADO}

Moricandia foetida Bourg. ex Coss.

ALMERÍA: Pulpí, 19-II-95, Lahora (MUB 46127). Cuevas de Almanzora, 30SXG0025, 15-X95, Lohora (MUB 46079). Alsodux, 24-III-85, Alcaraz (MUB 31114). Alsodux, 24-III-86, Alcaraz (MUB 16153). Tabernas, 30SVF58, 27-II-83, Alcaraz, (MUB 10177). Cuevas de Almanzora, 8 IV-90, sin recolector (MUB 33187). MiniHollywood, Tabernas, 21-IV-97. Sánchez Gómez et al. (MUB 49753).

Moricandia moricandioides (Boiss.) Heywood subsp. moricandioides

ALICANTE: Maigmó, 30SYH0462, $650 \mathrm{~m}$. 20-IV-85, De la Torre (MUB 19938). Maigmó, 30SYH0662, 500 m, 20-IV-86, De la Torre (MUB 14965). Maigmó, 30SYH0461, 500 m, 20-IV-85, De la Torre (MUB 19937). MURCIA: La Rogativa, Moratalla, 30SWH6720, 1200 m, 18-VI-81, Selma (MUB 12429, MUB 42796). Sierra Espuña, Alhama de Murcia, 30SXG3786, $200 \mathrm{~m}, 1$-IV-85, Robledo (MUB 38759). Sierra de Pajares, Moratalla, 30SXH1146, 420 m, 22-III-80, Lozano \& Alcaraz (MUB 3650). Moratalla, 30SWH9426, 1000 m, 14V-83, Selma (MUB 42795). Embalse de 
Valdeinfierno, Lorca, IV-98, Hernández \& Coy (MUB 49754).

Moricandia moricandioides subsp.pseudo-foetida Sánchez Gómez, Carrión Vilches, Hernández \&

J. Guerra

MURCIA: Puerto del Garruchal, Murcia, 30SXH70,120 m, 20-III-01, Sánchez Gómez, Hernández \& Jiménez (MGC 49455). Puerto del Garruchal, Murcia, 30SXH70,120 m, 5-VI-00, Carrillo \& Jiménez (MGC 49456). Puerto del Garruchal, Sierra de la Cresta, Murcia, 30SXH7002. 100 m, 7-III-81, Alcaraz (MUB 5603).

\section{BIBLIOGRAFÍA}

BOLÓS, A. -1948- El género Moricandia en la Península Ibérica. Anales Jard. Bot. Madrid 6: 451-461.
HEYWOOD, V. H. -1964- Moricandia DC. En Tutin, T.G. et al. (eds.). Flora Europaea, Vol. I. Cambridge University Press. Cambridge: 334. SÁNCHEZ GÓMEZ, P., J. GUERRA, E. COY, A. HERNÁNDEZ, S. FERNÁNDEZ y A. F. CARRILLO. 1998. Flora de Murcia. Claves de identificación e iconografía de plantas vasculares. Editorial DM. Murcia.

SOBRINO, E. -1993- Moricandia DC. En Castroviejo, S. et al. (eds.). Flora iberica, Vol. IV. C.S.I.C. Madrid: 337-344.

SOBRINO, E. -1997- Infertility in the genus Moricandia DC. Lagascalia 19: 839-844.

Aceptado para su publicación en octubre de 2001

Dirección de los autores. Departamento de Biología Vegetal (Área de Botánica). Universidad de Murcia. Campus de Espinardo s/n. E-30100 Murcia. E-mail: psgomez@um.es.

\title{
100. NOTA COROLÓGICA SOBRE LA FLORA DE EXTREMADURA (ESPAÑA)
}

\author{
Francisco María VÁZQUEZ PARDO, Soledad RAMOS MAQUEDA, \\ Esperanza DONCEL PÉREZ, Enrique BALBUENA GUTIÉRREZ, \\ José Antonio CASASOLA HERRERA y José BLANCO SALAS
}

\author{
Floristic records for the Flora of Extremadura (Spain) \\ Palabras clave. Badajoz, Cáceres, Corología, Extremadura, Flora.
}

Key words. Badajoz, Cáceres, Corology, Extremadura, Flora.

Con este trabajo se amplía el número de taxones conocidos para la flora de la región extremeña en 7 nuevos casos no citados hasta la fecha e incrementa el área de distribución en otros 3 de los que se tenían escasas referencias sobre su presencia en la flora regional. 